\title{
ARTICLE \\ Managing New PV Plant Connection to Available Grids to Stay within Standard Limits with a Case Study
}

\section{Ehsan Najafi $^{1^{*}}$ Amin Mirzaei $^{2}$ Mahdi Rezvanyvardom ${ }^{2}$ Mahdi Zolfaghar $^{1}$}

1. Faculty of electrical and computer engineering, Qom university of technology, Iran

2. Department of Electrical Engineering, Faculty of Engineering, Arak University, Arak, Iran

\begin{tabular}{l}
\hline ARTICLE INFO \\
\hline Article history \\
Received: 30 May 2021 \\
Accepted: 16 June 2021 \\
Published Online: 30 June 2021 \\
\hline Keywords: \\
PV plant \\
Grid connection study \\
Voltage profile \\
Solar energy \\
STATCOM
\end{tabular}

\section{Introduction}

PV plants are spreading all over the world due to their clean performance. In addition, their cost has been considerably reduced and their construction is straight forward. However, they impose some challenges to the electric grids that shall be well analyzed before safe connection. The analysis is performed by modelling available electric network within suitable simulation software and performing load flow analysis ${ }^{[1]}$. One of the important impacts of the plants is on the voltage characteristics of the grid usually leading to over voltages ${ }^{[2]}$. Although this experience does not occur in all cases, it shall be well considered before a plant connection to avoid any voltage disturbances ${ }^{[3]}$. The values of allowable

\section{ABSTRACT}

PV plants are increasing all over the world and they are becoming a distinct part of electric grids. Due to abundance of solar irradiation and almost constant amount of it in certain geographical latitudes, selection of proper capacity of PV plants depends mostly on available places for the site. In this paper, important measures for safe connection of a PV plant in terms of voltage requirements are addressed and several guidelines are introduced for this purpose. In addition, simulation results are included to prove some of the mentioned suggestions. A general algorithm is finally proposed to show the directions for safe connection of PV plants.

voltage limits are based on standards such as EN50160 or national grid codes in each country. The acceptable voltage levels in Iran national grid code are the same as ANSI C84.1-2016 and IEC 60038-2009 standards ${ }^{[4]}$ which are $\pm 5 \%$ for medium voltage levels while these levels are $\pm 10 \%$ in EN50160 ${ }^{[3]}$. If the voltage exceeds the standard limits during initial calculations several measures can be adopted. At first, main substation tap changer can be adjusted so that the overall voltage profile of the feeders stay within the limits ${ }^{[5]}$. While tap changing is a regular practice during normal operation of grids, it shall be well manipulated based on the performance of the PV plant ${ }^{[6]}$.

Another approach for solving the voltage disturbance in the presence of the plants is achieved by selection of a

*Corresponding Author:

Ehsan Najafi,

Faculty of electrical and computer engineering, Qom university of technology, Iran;

Email:najafi@qut.ac.ir 
suitable capacity for the PV plant. This value is a pivotal issue of the PV plant and is primarily selected based on the financial conditions of the project and the amount of capital investment. According to available statistics, each megawatt overall installation of the plants usually costs about 600,000 US $\$$ and this value is subject to change based on the size of the project, used technology and site conditions (such as the leveling and ground material). Based on the determined power of the plant, some grid studies are performed to investigate if the plant can be connected safely to the grid. One of the most important concerns for plant proper connection is voltage profile after connection that can be derived from load flow analysis. This calculation that is performed by wellknown software such as ETAP or DIgSILENT, illustrates the amount of grid parameters such as voltage and line loss in different conditions. Based on standards, amount of voltage rise due to the plant shall not exceed $5 \%$ above normal value. This issue shall be investigated during two extreme conditions of low and high load condition. In low power consumption, the PV power mostly goes through the main feeder and is not consumed in the connected line. This is a limiting factor for PV plants and will cause an excessive voltage at the point of common coupling (PCC).

The other method for solving the problem of voltage disturbance is by reactive power compensation. The $\mathrm{PV}$ inverters are capable of producing reactive power at the expense of active power compromise. Therefore, they can be adjusted to consume reactive power (as an inductor) to reduce the voltage at PCC ${ }^{[7]}$. In order to improve this method, a battery energy storage system (BESS) can be also used to provide required reactive together with active power during compensation periods. However, this approach needs a coordinated power compensation algorithm that adds to the complexity of the project ${ }^{[4]}$. Another approach to use reactive power for voltage compensation utilizes extra devices such as SVC or STATCOM ${ }^{[8]}$. STATCOM is useful to mitigate other power quality disturbances such as voltage sag and swell during faults such short circuit ${ }^{[9]}$. However, they are costly and complex since they need several numerical and control blocks to measure and manipulate the voltage levels ${ }^{[10]}$.

In order to increase this limit practically in Iran, PV plants above $1 \mathrm{MW}$ are allowed to directly be connected to the main distribution substation via a dedicated feeder. While this is an optional scenario for plants with rated power less than $7 \mathrm{MW}$, it is compulsory for plants larger than $7 \mathrm{MW}$. All these measures are defined to make it possible for a plant to be connected correctly to the grid without any violation of standards. However, direct connection to the substation causes additional cost for the project including a dedicated feeder as well as a direct line to the substation. Therefore, PV plants are limited to the power that are allowed to be connected to the available grid due to financial concerns. It is worth mentioning that allocation of the site near the main substation will greatly enhance the connection characteristics via available grid but usually it is not feasible due to lack of available free places for the plant. On the other hand, high loading of a feeder leads to voltage drop in the neighboring feeders that may violate voltage standards and cause the voltages at the end of feeders to become less than limits. Therefore, this scenario shall also be well analyzed and investigated to avoid any problem during site connection.

In order to investigate if a plant is allowed to be connected to available grid several scenarios shall be assessed. At first, the voltage profile of the feeder that is supposed to be connected to the plant shall be simulated at the presence of the plant in low load conditions to check if the voltage does not increase from the limits (5\%). Another critical case is during high load conditions when the substation voltage is increased to mitigate voltage drop of the feeders at the end of lines. In this case, other feeders shall be also analyzed to check if other lines face low voltages beyond the limits at the end of their lines. These two concerns shall be well addressed before successful connection of a new PV plant to an available grid.

In this article a PV plant is investigated and its effect on the grid parameters is assessed. In order to investigate this case, a real network data in Iran is used and the simulation is done by DIgSILENT. In this case, a 5 MWP PV plant is intended to be connected to an available line and this article investigates the effect of the connection on the grid in terms of power loss and voltage magnitude. It shall be noted that the capacity of a PV plant is calculated based on the installed PV panels that is DC power at standard test conditions (STC). The maximum AC output power that is injected to the grid is about $10 \%$ less than this amount accounting for several losses such as panel loss (light induced degradation (LID), mismatch, soiling, etc.), inverter loss, cable loss, transformer loss, etc.

\section{Grid Study}

The plant is located almost at the middle of the line (i.e. 17 kilometers from the substation) that is around 30 kilometers. The overall single line diagram of the feeder together with another neighboring feeder is illustrated in the following figure. In this diagram, the main feeder for connecting the plant is named as feeder 1 and is in cyan while the other feeder is named feeder 2 and is in red. Both feeders are radial and their normal voltage is $20 \mathrm{kv}$. 
The transformer in the substation also has 5 steps in tap changing and can change the output voltage within $-5 \%$ and $5 \%$.

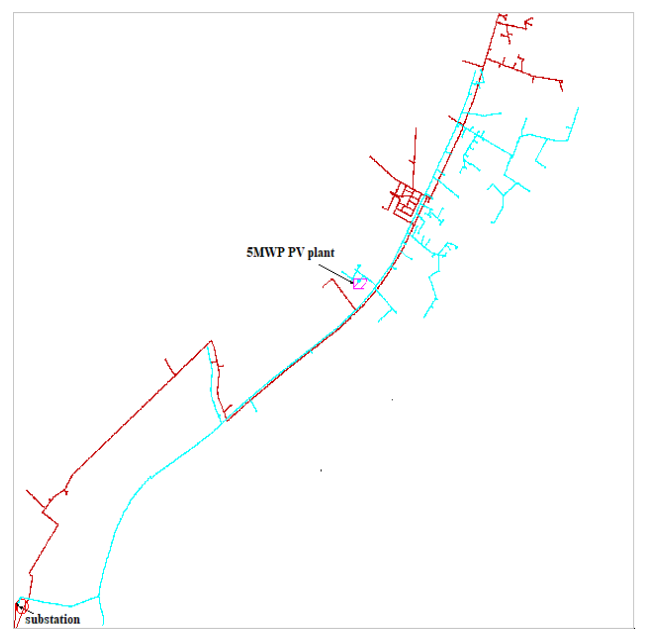

Figure 1. single line diagram of the analyzed grid in presence of 5MW PV plant connected to feeder1 (in cyan) and a neighboring feeder (feeder 2 in red)

It shall be assessed whether the plant negatively affects the voltage profile of the grid or not. At first, the performance of the plant is analyzed during low load conditions that usually happens during cold seasons. Based on the load profile in winter, $1 \mathrm{MW}$ is the lowest load during mid-day while the solar plant creates the highest power. So, analysis is carried out at this occasion and the result is illustrated as in Figure 2. According to the voltage profile, the line does not face any problem and the voltages are within standard limits. It is pertinent to note that the substation voltage is also set at its nominal value $(1 \mathrm{pu})$ to control the overall voltage profile of other feeders.

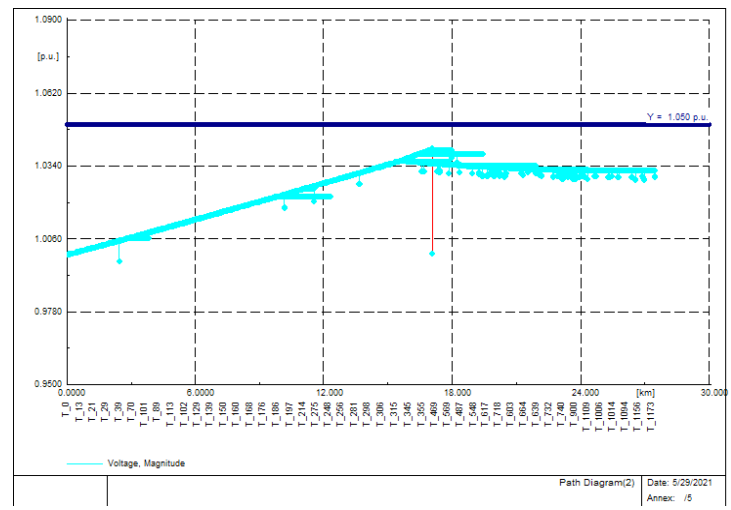

Figure 2. voltage profile of feeder 1 in presence of PV plant in low load condition

In order to investigate the effect of the plant on other feeders, another feeder which takes $1.74 \mathrm{MW}$ at the moment (feeder 2) is also investigated and its voltage profile is depicted in Figure 3. Based on the load profile, this result shows that the plant does not negatively affect other neighboring feeders. Another simulation is also performed to find maximum power of the other feeder for standard operation of it and the result was 2.9 MW. Therefore, there is a large margin for the load of feeder 2 to change. It is also pertinent to mention that any other set points in substation voltage will violate voltage limits either in feeder 1 or in feeder 2 . So, the accurate selection of set points in presence of plants is of the utmost importance.

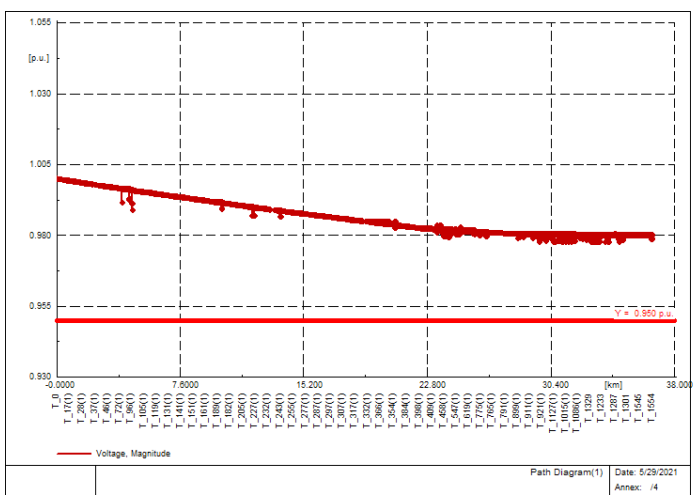

Figure 3. voltage profile of feeder 2 in low load conditions

Another concern for connecting the plant is in high load conditions. Therefore, the performance of the plant is also assessed during feeder high load status. In this case the substation voltage is usually set above nominal voltage to compensate line voltage drop alongside the line. Nonetheless, the plant may cause over voltage conditions beyond $5 \%$ that is not allowed by the standards. The voltage profile of the feeder is simulated and shown in Figure 4. In this case, the load of the feeder is approximately $5.5 \mathrm{MW}$ and the substation voltage is set to $1.025 \mathrm{pu}$ by the transformer tap changer. According to the results, the voltage profile is within acceptable limits and the highest value that is near the substation is $1.025 \mathrm{pu}$.

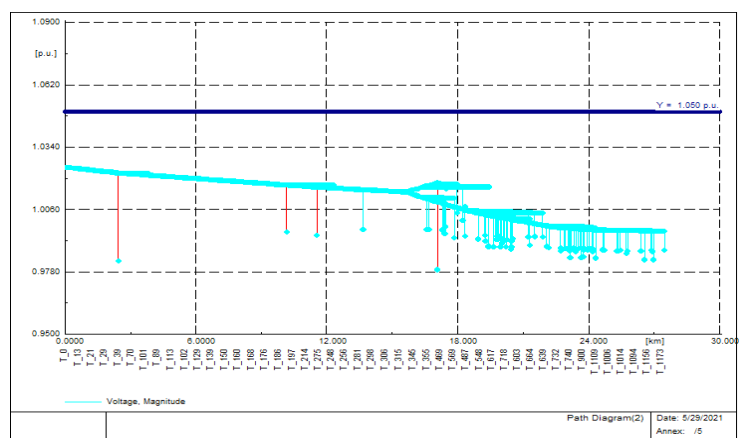

Figure 4. voltage profile of feeder 1 in presence of PV plant in high load condition 
To investigate the effect of the plant on other feeders, another simulation is done and the load for feeder 2 at the same time that feeder 1 is in high load is 1.13 . The voltage profile shows that this condition does not cause the feeder to have voltage levels beyond the limits. It is worth mentioning that due to the tap changer step limits (each step changes $2.5 \%$ ) if the load of the feeder exceeded $4 \mathrm{MW}$ that is far larger than the available loading, the voltage profile would drop beyond 5\% limit and would not be acceptable. On the other hand, increasing voltage level at the substation would lead to over voltages at PV plant bus. So, the correct selection of the tap is vital and all the loading conditions shall be considered and analyzed before final decision for the plant connection.

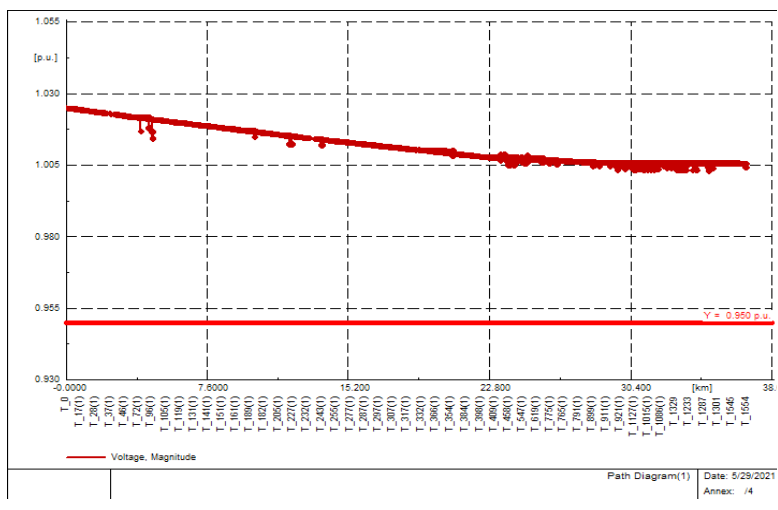

Figure 5. voltage profile of feeder 2 in high load conditions

One of the advantages of connecting a PV plant in the middle of a line is reduction of power loss along the line. This happens especially during high load condition since the power does not need to transfer from the beginning of the line to the end to reach the consumers. The power can be supplied by the PV plant for the loads at the end of the line and near the PV plant. Therefore, the generation and consumption of electric power become close to each other leading to decrease in power loss over the line. This issue is also assessed and the results show that $452 \mathrm{KW}$ loss without the plant culminated in $213 \mathrm{KW}$ loss (about $47 \%$ less) due to the PV plant operation.

\section{PV Plant Connection Algorithm}

In order to generalize this approach, the following algorithm is developed. Based on the flowchart, the PV plant can be sized and selected properly in order not to violate grid voltage limits. According to the above discussion, low load in the grid shall be analyzed primarily. If any voltage violation occurs (increase from defined limits at the point of connection), tap changing shall be exercised so that voltage profile be kept within standard limits. If the practice does not answer, the PV plant capacity shall be reduced so that at the point of connection the grid voltage becomes less than the maximum limit.

Finally, the voltage profile is again found in the high load conditions. In this case, other feeders may face low voltage at the end of their network. On one hand the substation voltage with changing the tap of transformer can not be increased as before due to the PV plant and its effect. On the other hand, other feeders may come across low voltage problems at the end of their lines. The simulation in high load, assesses if the PV plant connection will culminate in reduction of voltage at the end of other feeders. If the simulation in this case does not show any abnormal conditions, then the PV plant can be safely connected to the grid. Otherwise some measures as discussed in the flowchart shall be taken to solve the problem.

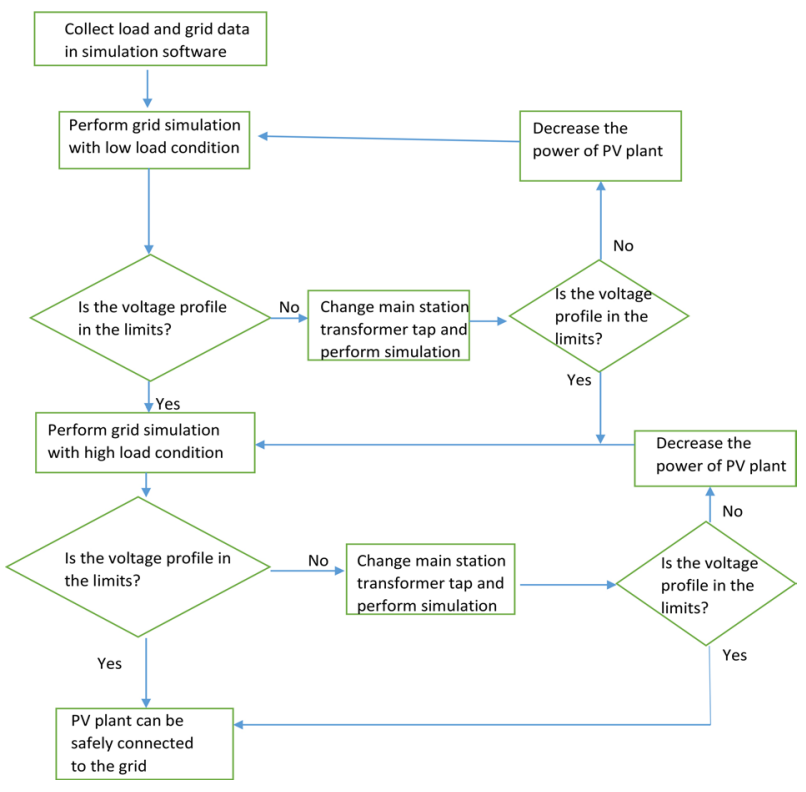

Figure 6. proposed algorithm for PV plant connection with considering voltage limitations

\section{Conclusions}

In this paper, an approach is proposed to manage connection of new plants to the grid. A PV plant connection study as a case study is discussed in the paper and its effect on neighboring feeder is also investigated. The paper illustrated that in certain conditions (tap position of the substation transformer) and high load and low load conditions, the plant can safely be connected to the available grid. This shows the importance of voltage management in the main substation in order to have safe operation of the PV plant. Finally, an algorithm is proposed to be followed for grid studies when a new PV 
plant is supposed to be connected to a grid.

\section{References}

[1] P. Jayakumar and P. Reji, "Modeling of power distribution feeder and analysis of small PV plant penetration in Kerala low voltage distribution system," in 2016 International Conference on Cogene-ration, Small Power Plants and District Energy (ICUE), 2016, pp. 1-6.

[2] S. Pukhrem, M. Conlon, and M. Basu, "Mitigation of over-voltage fluctuation in medium voltage (MV) distribution feeder line with high PV plant penetration," in 2015 50th International Universities Power Engineering Conference (UPEC), 2015, pp. 1-5.

[3] I. Ramljak and D. Bago, "Influence of PV plant connection on voltage quality parameters considering connection point in distribution grid," in 2018 First International Colloquium on Smart Grid Metrology (SmaGriMet), 2018, pp. 1-5.

[4] Z. Zhang, Y. Mishra, C. Dou, D. Yue, B. Zhang, and Y. C. Tian, "Steady-State Voltage Regulation With Reduced Photovoltaic Power Curtailment," IEEE Journal of Photovoltaics, vol. 10, pp. 1853-1863, 2020.

[5] Y. Agalgaonkar, B. C. Pal, and R. A. Jabr, "Distribution voltage control considering the impact of PV generation on tap changers and autonomous regulators," in 2014 IEEE PES General Meeting | Conference \& Exposition, 2014, pp. 1-1.

[6] F. Bai, R. Yan, T. K. Saha, and D. Eghbal, "A New Remote Tap Position Estimation Approach for OpenDelta Step-Voltage Regulator in a Photovoltaic Integrated Distribution Network," IEEE Transactions on Power Systems, vol. 33, pp. 4433-4443, 2018.

[7] H. Zhang, C. Xia, P. Peng, N. Chen, and B. Gao, "Research on the Voltage Regulation Strategy of Photovoltaic Power Plant," in 2018 China International Conference on Electricity Distribution (CICED), 2018, pp. 1620-1624.

[8] E. Najafi and A. H. M. Yatim, "A D-STATCOM based on Goertzel algorithm for sag detection and a novel current mode controller," in 2010 5th IEEE Conference on Industrial Electronics and Applications, 2010, pp. 1006-1011.

[9] M. Moghbel and M. A. S. Masoum, "D-STATCOM based on hysteresis current control to improve voltage profile of distribution systems with PV solar power," in 2016 Australasian Universities Power Engineering Conference (AUPEC), 2016, pp. 1-5.

[10] E. Najafi and A. H. M. Yatim, "A novel current mode controller for a static compensator utilizing Goertzel algorithm to mitigate voltage sags," Energy Conversion and Management, vol. 52, pp. 19992008, 2011. 